\title{
Bioluminescent indicator applicable to membrane voltage recording in various excitable cell types
}

Shigenori Inagaki, Takeharu Nagai

Shigenori Inagaki, Takeharu Nagai, "Bioluminescent indicator applicable to membrane voltage recording in various excitable cell types," Proc. SPIE 10251, Biomedical Imaging and Sensing Conference, 1025102 (18 April 2017); doi: 10.1117/12.2267502

Event: SPIE Technologies and Applications of Structured Light, 2017, Yokohama, Japan 


\title{
Bioluminescent indicator applicable to membrane voltage recording in various excitable cell types
}

\author{
Shigenori Inagaki ${ }^{\mathrm{a}}$, Takeharu Nagai ${ }^{\mathrm{a}, \mathrm{b}}$ \\ ${ }^{a}$ Graduate School of Frontier Biosciences, Osaka University, 1-3 Yamadaoka, Suita, Osaka 565-0871, \\ Japan \\ b The Institute of Scientific and Industrial Research, Osaka University, 8-1 Mihogaoka, Ibaraki, \\ Osaka 567-0047, Japan
}

\begin{abstract}
Here, we report a world-first bioluminescent indicator for membrane voltage, LOTUS-V. Since it is able to reveal voltage dynamics without external light source, LOTUS-V serves high contrast voltage imaging free from the effect of autofluorescence, suggesting its great versatility in the wide range of bioscience.
\end{abstract}

Keywords: bioluminescence imaging, voltage indicator, excitable cells, cardiomyocytes

\section{Introduction}

Genetically encoded voltage indicators (GEVIs) are promising tools for directly imaging voltage dynamics and/or spiking activity of genetically defined cell types ${ }^{1-3}$. However, the strong excitation light, which is usually required for voltage imaging causes severe autofluorescence especially in the tissue sample. Furthermore, it often poses problems such as photobleaching and phototoxicity, the latter being particularly limiting in fragile cells ${ }^{4,5}$.

The best way to avoid these problems is to perform voltage imaging that doesn't require excitation light, such as by using bioluminescent proteins. We previously developed the Nanolantern series ${ }^{6}$, bioluminescent proteins consisting of a Renilla luciferase (RLuc) variant and the yellow fluorescent protein (Venus) ${ }^{7}$, in which the bioluminescence intensity is enhanced by Förster resonance energy transfer (FRET). Then, we applied the Nano-lantern to develop several indicators for biological elements including $\mathrm{Ca}^{2+}$ and ATP, thereby succeeded $\mathrm{Ca}^{2+}$ imaging with the optical stimulation of Channelrhodopsin-2, and ATP imaging during photosynthesis in a plant leaf. In line with this trend, we expanded the application of bioluminescent indicators to voltage imaging.

\section{Material and Methods}

\section{Development of a bioluminescent voltage indicator}

The design of the bioluminescent GEVI (bGEVI) was inspired with the FRET-based GEVIs such as VSFP BF1.2 and Mermaid $2^{8,9}$. To develop a bright bGEVI, we used NanoLuc which produces approximately 150-times the luminescence of
RLuc $^{10}$ and Venus as a FRET donor and acceptor, respectively. Similar to VSFP BF1.2 and Mermaid2, the working principle of

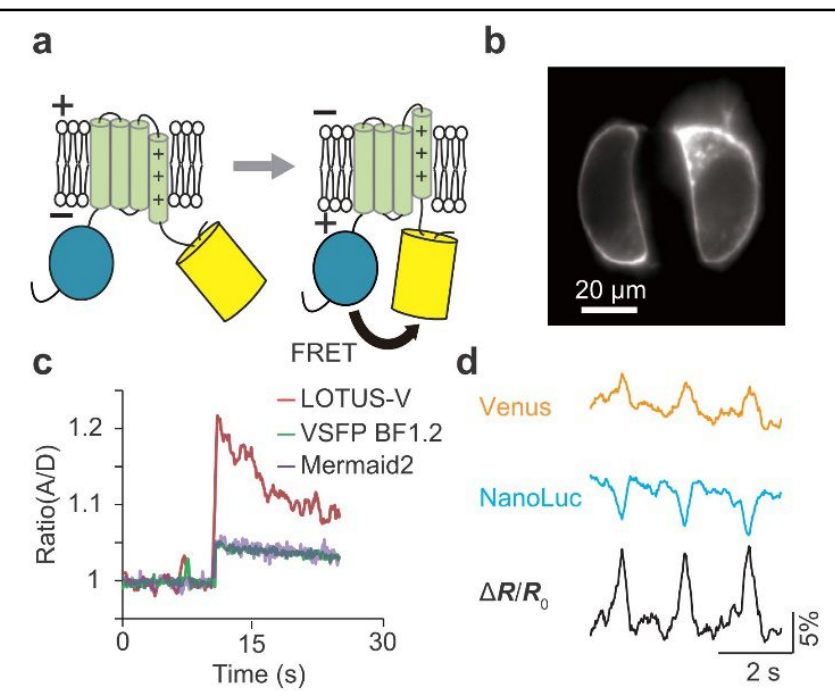

Figure. (a) Schematic diagram illustrating the voltage sensing mechanism of LOTUS-V. Depolarization of the membrane voltage induces a structural change in the VSD (green), thereby increasing the FRET efficiency between NanoLuc (blue) and Venus (yellow). (b) Bioluminescence image of LOTUS-V expressing in GH3 cells. (c) Time series of the ratio value of LOTUS-V (red), VSFP BF1.2 (green) and Mermaid2 (purple) in $\mathrm{GH} 3$ cells. $\mathrm{KCl}$ solution was applied at $10 \mathrm{~min}$. (d) Representative optical responses of Venus (yellow), NanoLuc (cyan), and its ratio change $\left(\Delta R / R_{0}\right.$; black) in response to hiPSCCMs contractions. 
our bGEVI is that a change in FRET is induced by the voltagedependent movement of the voltage sensitive domain (VSD(R217Q)) of Ci-VSP ${ }^{11}$ on the plasma membrane (Fig. a). Finally, we designated this bGEVI as LOTUS-V (Luminescent Optical Tool for Universal Sensing of Voltage).

\section{Results and Discussion}

\section{Comparison with other fluorescent GEVIs}

When we added the bioluminescent substrate, furimazine to the imaging medium, intense bioluminescence from rat pituitary epithelial-like tumor (GH3) cells was observed. (Fig. b). Comparing with VSFP BF1.2 or Mermaid2, the signal change in LOTUS-V upon $\mathrm{KCl}$-induced depolarization was more than four times that seen with them (Fig. c).

\section{Voltage imaging in hiPSC-CMs}

To examine the compatibility with excitable cell types that move we chose an in-vitro cardiomyocyte model. Then, we expressed LOTUS-V in cardiomyocytes derived from human induced pluripotent stem cells (hiPSC-CMs) via lentivirus gene expression system. During spontaneous contraction, a reciprocal change in the NanoLuc and Venus intensities was observed. The increase in the emission ratio value suggested a precise reflection of the action potential in cardiomyocytes (Fig. d).

\section{Conclusion}

Biouminescence imaging has been generally considered too dim for voltage imaging that requires a fast frame rate acquisition. To overcome this problem, we developed a bright bGEVI using NanoLuc, which is the brightest bioluminescent protein, and demonstrated that it was applicable for voltage imaging. As for in-vitro cardiomyocytes model, LOTUS-V mitigated the effect of motion artifact owing to ratiometric measurement and successfully captured the cardiac action potential. Collectively, bioluminescence imaging has several advantages over fluorescence imaging. LOTUS-V makes voltage imaging applicable to situations that are difficult to monitor with fluorescent GEVIs.

\section{References}

[1] Siegel, M. S.., Isacoff, E. Y., "Probe of Membrane Voltage," 735-741 (1997).

[2] Dimitrov, D., He, Y., Mutoh, H., Baker, B. J., Cohen, L., Akemann, W.., Knöpfel, T., "Engineering and Characterization of an Enhanced Fluorescent Protein Voltage Sensor," PLoS One 2(5), e440 (2007).

[3] Kralj, J., Douglass, A., Hochbaum, D., Maclaurin, D.., Cohen, A., "Optical recording of action potentials in mammalian neurons using a microbial rhodopsin," Nat Meth 9(1), 90-95, Nature Publishing Group, a division of Macmillan Publishers Limited. All Rights Reserved. (2012).

[4] Leyton-Mange, J. S., Mills, R. W., Macri, V. S., Jang, M. Y., Butte, F. N., Ellinor, P. T.., Milan, D. J., "Rapid cellular phenotyping of human pluripotent stem cellderived cardiomyocytes using a genetically encoded fluorescent voltage sensor," Stem Cell Reports 2(2), 163-170, The Authors (2014).

[5] Shinnawi, R., Huber, I., Maizels, L., Shaheen, N., Gepstein, A., Arbel, G., Tijsen, A. J.., Gepstein, L., "Monitoring Human-Induced Pluripotent Stem CellDerived Cardiomyocytes with Genetically Encoded Calcium and Voltage Fluorescent Reporters," Stem Cell Reports 5(4), 582-596, The Authors (2015).

[6] Saito, K., Chang, Y.-F., Horikawa, K., Hatsugai, N., Higuchi, Y., Hashida, M., Yoshida, Y., Matsuda, T., Arai, Y., et al., "Luminescent proteins for high-speed single-cell and whole-body imaging.," Nat. Commun. 3(May), 1262, Nature Publishing Group (2012).

[7] Nagai, T., Ibata, K., Park, E. S., Kubota, M., Mikoshiba, K.., Miyawaki, A., "A variant of yellow fluorescent protein with fast and efficient maturation for cellbiological applications.," Nat. Biotechnol. 20(1), 87-90 (2002).

[8] Akemann, W., Mutoh, H., Perron, A., Park, Y. K., Iwamoto, Y.., Knöpfel, T., "Imaging neural circuit dynamics with a voltage-sensitive fluorescent protein.," J. Neurophysiol. 108(8), 2323-2337 (2012).

[9] Tsutsui, H., Jinno, Y., Tomita, A., Niino, Y., Yamada, Y., Mikoshiba, K., Miyawaki, A.., Okamura, Y., "Improved detection of electrical activity with a voltage probe based on a voltage-sensing phosphatase.," J. Physiol. 591(Pt 18), 4427-4437 (2013).

[10] Hall, M. P., Unch, J., Binkowski, B. F., Valley, M. P., Butler, B. L., Wood, G., Otto, P., Zimmerman, K., Vidugiris, G., et al., "Engineered Luciferase Reporter from a Deep Sea Shrimp Utilizing a Novel Imidazopyrazinone Substrate.”

[11] Murata, Y., Iwasaki, H., Sasaki, M., Inaba, K.., Okamura, Y., "Phosphoinositide phosphatase activity coupled to an intrinsic voltage sensor.," Nature 435(7046), 1239-1243 (2005). 